\title{
Lid-Driven Cavity For Mantle Convection Modelling Using Lattice Boltzmann Method
}

\author{
Fredy Tantri ${ }^{1 *}$, Umar Fauzi ${ }^{1}$, Fourier D. E. Latief ${ }^{1}$ \\ ${ }^{1}$ Physics of Earth and Complex Systems, Faculty of Mathematics and Natural Sciences, Institut Teknologi Bandung, \\ Jalan Ganesha 10, Bandung 40132, Indonesia
}

( Received: September 28,2021, Revised: October 31,2021, Accepted: November 14,2021 )

\begin{abstract}
The Lattice Boltzmann Method is one of the computational fluid dynamics methods that can be applied to simulate fluid based on the microscopic and kinetic theory of gases. In this study, earth mantle convection is simulated by combining the concept of lid-driven cavity simulation and natural convection using the Lattice Boltzmann method in a two-dimensional system (D2Q9). The results of the lid-driven cavity and natural convection simulation are comparable to previous works. This study shows that at a certain lid velocity, the direction of the moving plume is changed. This earth mantle convection simulation will give better and more reliable results by considering more complicated boundary conditions and adequate simulation systems.
\end{abstract}

Keywords: fluid dynamics, Lattice Boltzmann Method, lid-driven cavity, earth mantle convection

\section{INTRODUCTION}

Simulating fluid flows remains a big challenge for scientists because of the unsolved complete NavierStokes equation in the complex geometry domain. Therefore, numerical methods are developed and used to overcome this problem. One of the current prospective methods is the lattice Boltzmann method (LBM). This method is based on microscopic models and kinetic equations, in contrast to the conventional method which is based on the discretization of continuous macroscopic equations. By developing simple kinetic equations, solving complex equations such as the Navier-Stokes equation, or reviewing individual particles as in molecular dynamics simulations can be avoided. Therefore, this method has been successfully applied in complex boundary conditions and interactions between fluids with different phases.

The LBM concept is derived from lattice gas automata, a kinetic model of discrete particles on a discrete lattice and time. The idea to utilize this kinetic equation was first carried out by Broadwell [1] to study one-dimensional wave propagation. In this model, space and time are still continuous. Furthermore, a model with discrete space and time was introduced by Hardy et al. (1976) to study the fluid transport properties [2]. Then, many fundamental concepts in constructing the NavierStokes equation from lattice gas automata were developed and elaborated [3-7].

The concept of a lattice gas assumes that each lattice can only be filled by a boolean variable ( 1 or $0)$. LBM replaces the concept of a boolean variable with a particle distribution function so that the motion of individual particles can be ignored. According to McNamara and Zanetti (1988), this step eliminates interference statistically [8]. The simplification of the collision operator was carried out by Higuera and Jimenez (1989) by assuming that the distribution function has a value close to the local equilibrium state [9]. This simple linear model of the collision operator utilizes the relaxation concept known as the Bhatnagar-Gross-Krook (BGK) collision operator [10]. Sterling and Chen (1996) showed that the Boltzmann lattice equation could be considered a finite difference discretization of the Boltzmann

* Corresponding author.

E-mail address: umarf@fi.itb.ac.id 
equation [11]. He and Luo proved that LBM could be derived from the kinetic theory of gases [6].

It was shown that this method was successfully applied in complex geometry as well as in multiphase interactions. The capability of this method challenges researchers to apply for simulating convection of the earth interior, since understanding the dynamics of earth interior is not yet satisfied due to its complexity and technology limitations.

Guo and Zhao (2005) studied convection heat transfer in porous media with a new distribution function to simulate the temperature field. The results show good agreement with the well-documented literature [12]. Wanga et al. (2013) used the MRT thermal LBM to simulate two flows in two dimensions of a cavity with differentially heated vertical walls and a rectangle heated from below [13].

Mora and Yuen (2017) presented a 2-D simulation of a fluid in a rectangular box that is heated from the bottom and cooled from above to mimic the regime of relevance for geodynamical problems using the LBM. They showed that the LBM has significant potential for simulating thermal convection and plume dynamics relevant to geodynamics with some limitations [14]. Using the enthalpy-based Lattice Boltzmann Method, Dai et al. (2018) simulated natural convection melting in a cavity heated from different sides. The cavity model in this study is heated on one side, and three other sides are adiabatic and have melting fluid [15].

Rui et al. (2020) applied the enthalpy-based lattice Boltzmann model combined with the pseudopotential Lattice Boltzmann model to trace the solidliquid interface for the natural convection melting in a square cavity [16]. Bourada et al. (2020) analyzed a problem of mixed convection numerically in a liddriven square cavity, in which the upper wall is movable and kept at constant cold temperature. In contrast, the cavity contains a porous obstacle placed on the bottom wall with a constant hot temperature [17].

It is shown that in many developed models, the tectonic plate and mantle are assumed as different layers that are moving interdependently [18]. This model will be chosen in this paper. The wellestablished method of simulating fluid flow in liddriven cavity problems and natural convection inspires us to simulate mantle convection by combining both phenomena. This idea is based on the resemblance between lid-driven cavity phenomenon and tectonic plate movement at earth's surface and natural convection phenomenon similar to a mantle's plume.

\section{MODEL AND ALGORITHM}

\section{Lattice Boltzmann Method}

The key of the Lattice Boltzmann Method is the following equation $[4,5]$ :

$$
\begin{aligned}
& f_{i}\left(r+c_{i} \Delta t, t+\Delta t\right)=f_{i}(r, t)+\frac{\Delta t}{\tau}\left[f_{i}^{e q}(r, t)-\right. \\
& \left.f_{i}(r, t)\right],
\end{aligned}
$$

where $f_{i}, f_{i}^{e q}$ and $\tau$ stand for the distribution function of $i$ direction, equilibrium distribution function, and relaxation time, respectively. The equation determines the final distribution function formed at the end of the relaxation time interval. Then, the equilibrium distribution function is obtained from normalized Maxwell distribution function with Taylor expansion [4,5]

$$
f=\frac{\rho}{\frac{2 \pi}{3}} e^{-\frac{3}{2}\left(c^{2}\right)}\left[\begin{array}{c}
1+3(\boldsymbol{c} \cdot \boldsymbol{u})-\frac{3}{2} u^{2} \\
+\frac{9}{2}(\boldsymbol{c} \cdot \boldsymbol{u})^{2}
\end{array}\right] .
$$

If it is discretized at two dimensional with nine directions (D2Q9), the equilibrium distribution function is $[4,5]$

$$
\begin{aligned}
& \quad f_{i}^{e q}(x, t)=w_{i} \rho(x, t)\left[1+3\left(\boldsymbol{c}_{\boldsymbol{i}} \cdot \boldsymbol{u}\right)-\frac{3}{2} u^{2}+\right. \\
& \left.\frac{9}{2}\left(\boldsymbol{c}_{\boldsymbol{i}} \cdot \boldsymbol{u}\right)^{2}\right] .
\end{aligned}
$$

where $c_{i}$ and $w_{i}$ stand for unit vector and weighting coefficient along the direction $i$ which has value as following [5]:

$$
\begin{array}{ll}
c_{0}=(0,0) & , \mathrm{w}_{0}=4 / 9 \\
c_{1}=(1,0) & , \mathrm{w}_{1}=1 / 9 \\
c_{2}=(0,2) & , \mathrm{w}_{2}=1 / 9 \\
c_{3}=(-1,0) & , \mathrm{w}_{3}=1 / 9 \\
c_{4}=(0,-1) & , \mathrm{w}_{4}=1 / 9 \\
c_{5}=(1,1) & , \mathrm{w}_{5}=1 / 36 \\
c_{6}=(-1,1) & , \mathrm{w}_{6}=1 / 36 \\
c_{7}=(-1,-1) & , \mathrm{w}_{7}=1 / 36 \\
c_{8}=(1,-1) & , \mathrm{w}_{8}=1 / 36
\end{array}
$$

\section{Lid-driven Cavity}

This model consists of three static walls and a moving lid with constant speed $U$ (Fig. 1). It is assumed that we use incompressible fluid and no-slip walls. Initially, the cavity is homogenous and filled by zerovelocity fluid. In this simulation, we used $128 \times 128$ lattices, speed of lid $U=0.1$, and viscosity $v=$ 0.128 . This setting yields a Reynold number by 100 (based on $R e=U * N / v$ where $N$ is the total number 
of lattices in the direction of $U$ ). The 2-dimensional lattice Boltzmann method. The so-called D2Q9 is applied in this paper. We obtained the data of horizontal velocity of the fluid along the vertical line at $X=x / N_{x}=64 / 128=0.5$, and vertical velocity along the horizontal line at $Y=0.5$.

$$
u=U, v=0
$$

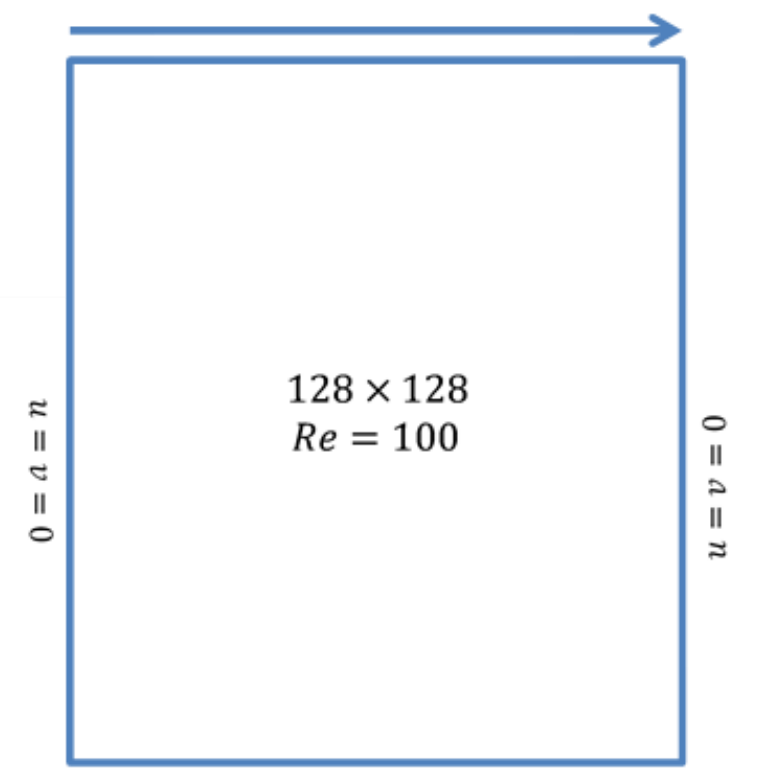

Fig. 1. Lid-driven cavity model.

The algorithm of the simulation is as follows. The simulation starts by defining several initial parameters, i.e., system size, cover rate, viscosity, wall position, and initial fluid density and rate in each grid. Calculation of distribution functions is then conducted using equations explained in the previous section. The distribution function will flow to its nearest neighbor lattice according to their respective directions. Bounce back limit conditions on system walls are applied. In the end, calculation of the density and velocity of the fluid at each lattice point in the system based on the updated distribution function is carried out. This process is then repeated until the expected result is reached. See Fig. 2 for the flowchart of the algorithm.

\section{Natural Convection}

The natural convection is illustrated as shown in Fig. 3 , where this model consists of a cold upper plate $\left(T_{1}=0\right)$ and hot bottom plate $\left(T_{0}=1\right)$. A periodic boundary condition connects the left and right sides of the system. The size of the system is 51 pixels in height and 102 pixels in width. A perturbation of $T=$ $T_{0}+T_{0} / 10$ is given on the center of the bottom plate.

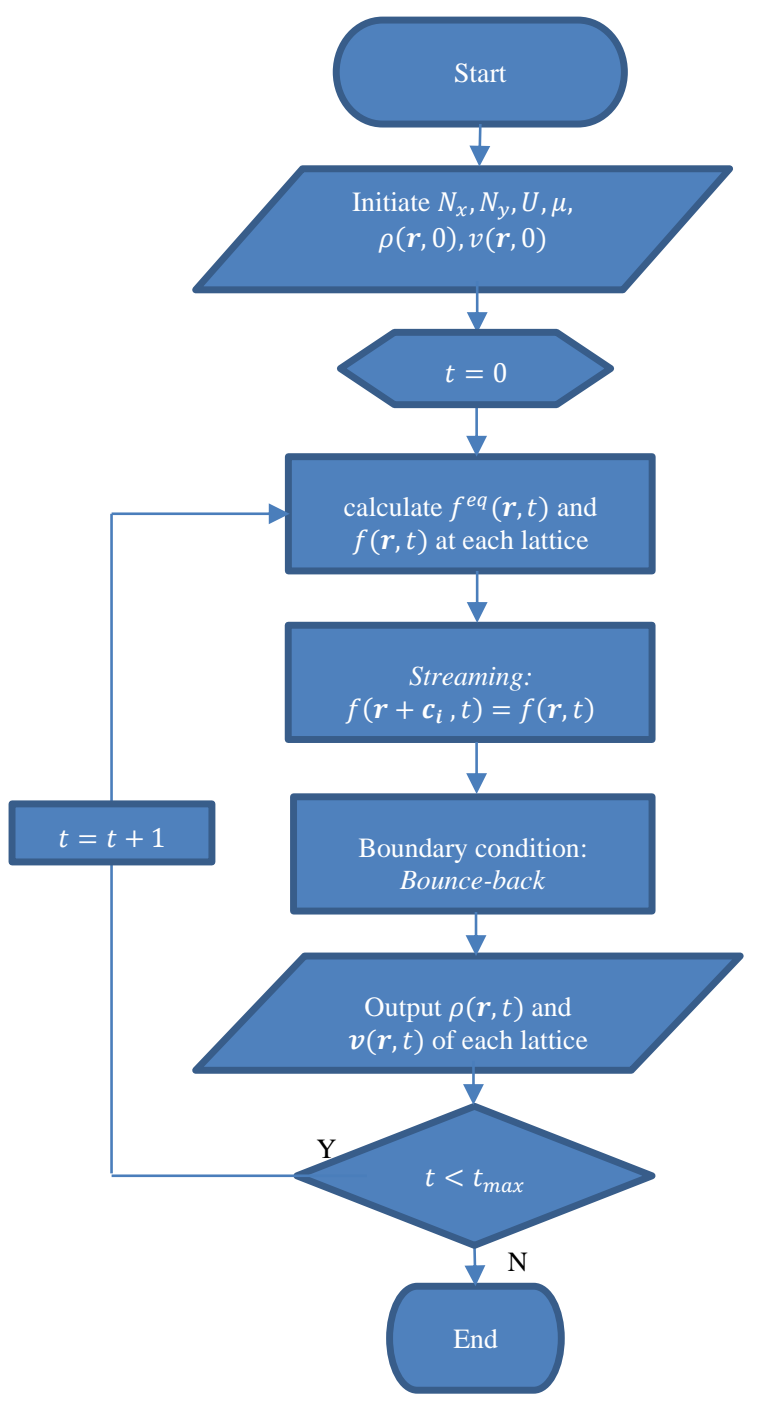

Fig. 2. Lid-driven cavity simulation flowchart.

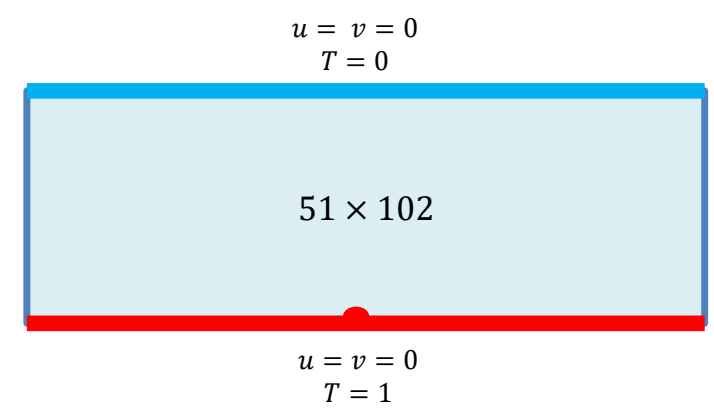

Fig. 3. Natural convection model.

The simulation algorithm of natural convection is shown in Fig. 4. It starts with inserting parameters, i.e., system size, viscosity, Prandtl number, Rayleigh number, wall position, temperature, density, and initial fluid rate in each lattice. It is then followed by calculating the temperature, density, and fluid velocity at each lattice point in the systems. The collision process follows the equation explained in the previous sections. Implementation of the bounceback boundary conditions on solid walls and periodic 
boundary conditions on the left and right sides of the system is carried out. Subsequently, the distribution function will flow to its nearest neighbor lattice according to their respective directions. Boundary conditions due to the influence of the temperature source on the top plate and bottom plate are applied. The process of calculating macroscopic quantities is conducted until the simulation time limit is reached.

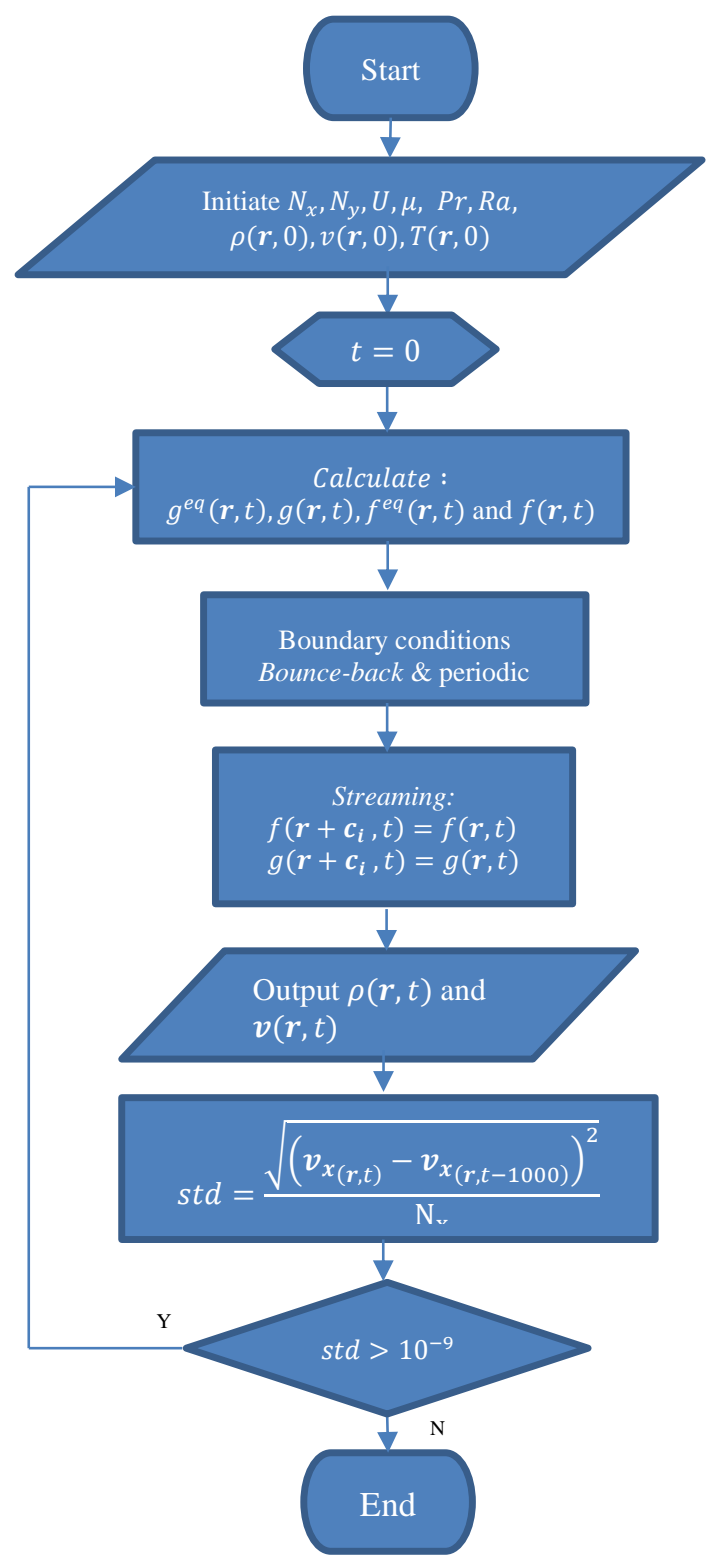

Fig. 4. Natural convection simulation flowchart.

\section{MANTLE CONVECTION}

This model is a combination of the lid-driven cavity and natural convection model. This model has a moving-cold-upper plate with velocity $U=0.1$ and a static-hot-bottom plate (Fig 5). Rayleigh number is $2,000,000$. The algorithm of this simulation is almost similar to the natural convection algorithm, and the only difference is the boundary condition for distribution function on the upper plate because the plate is moving like the lid in a lid-driven cavity problem.

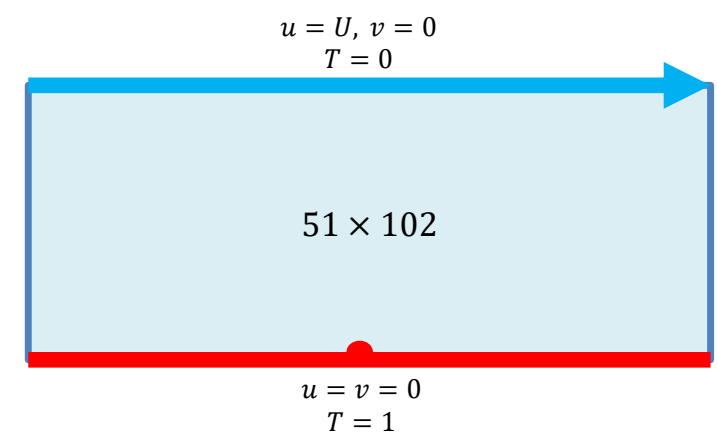

Fig. 5. Mantle convection model.

\section{RESULTS AND DISCUSSION}

\section{Lid-driven Cavity}

The graph of the horizontal velocity of the fluid along the vertical line at $X=0.5$ and vertical velocity along the horizontal line at $Y=0.5$ is shown in Fig. 6 and 7 , respectively. The data is taken at the $40,000^{\text {th }}$ time step.

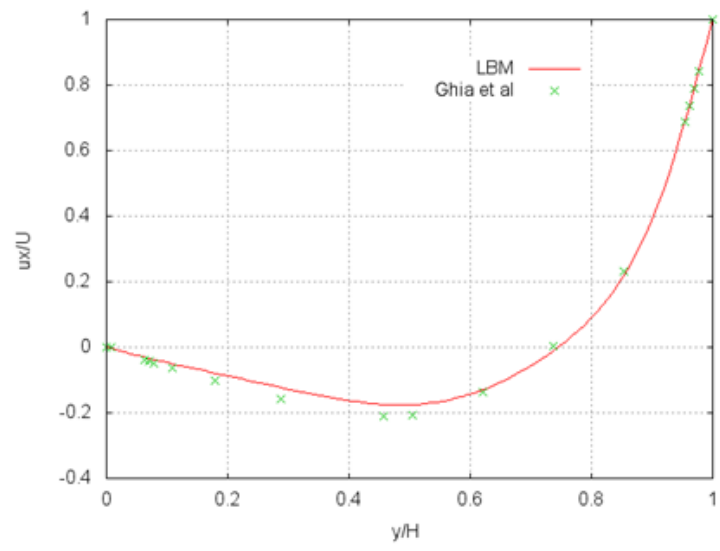

Fig. 6. Horizontal velocity plot using LBM and plot by Ghia et al. [19].

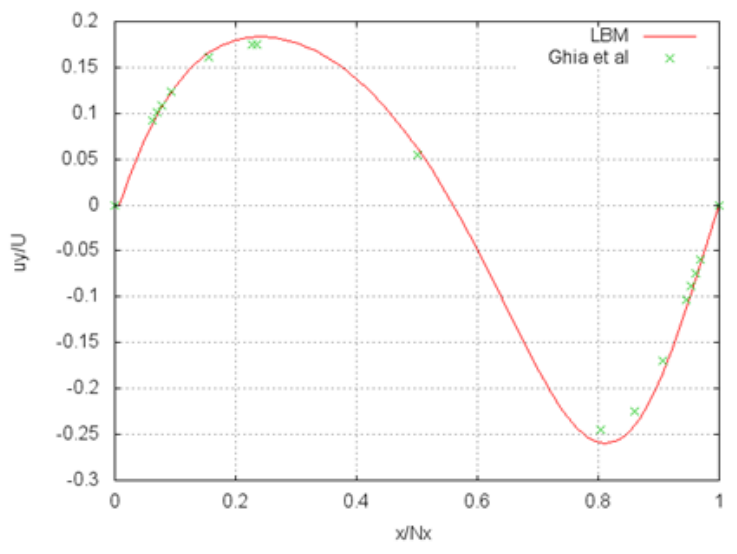

Fig. 7. Vertical velocity plot using LBM and plot by Ghia et al. [19] 
By comparing it to the result from Ghia et al. [19], the RMS error is only about $0.34 \%$ for horizontal velocity and $0.81 \%$ for vertical velocity. Our reference uses the Navier-Stokes equation and finite difference in solving the lid-driven cavity problem [4].
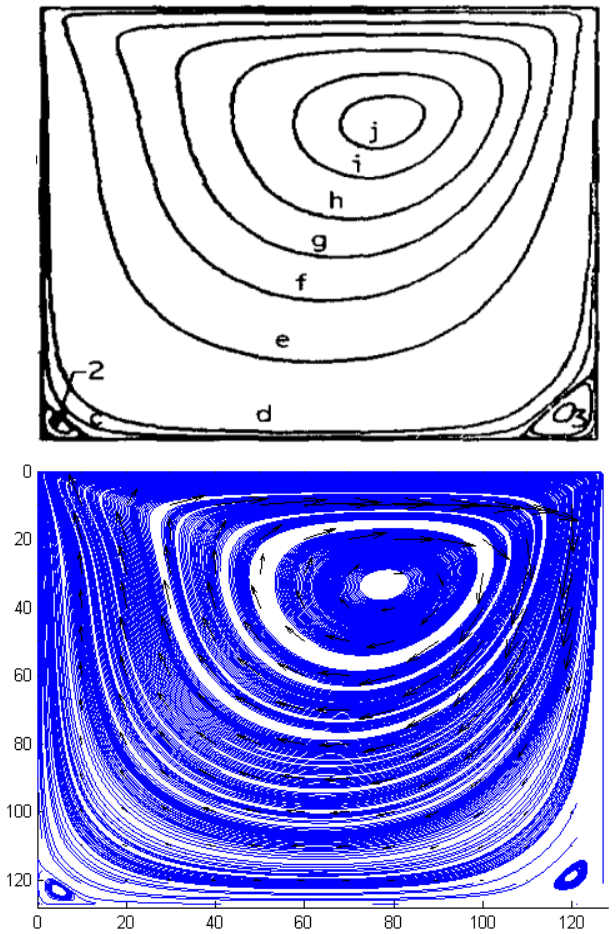

Fig. 8. Top: streamline in the cavity by Ghia et al. [19], bottom: streamline with vector velocity in the cavity by LBM.

Analyzing the graphs above, we can predict that the moving lid creates a vortex inside the cavity. This claim is proven when we plot the streamline of the fluid (Fig. 8 bottom). We also plot the velocity vector to see the direction of the moving fluid.

\section{Natural Convection}

Analytically, natural convection occurs at a Rayleigh number of 1707.762 [20]. In this simulation, we observed that the critical Rayleigh number is 1728 . This shows that the error is only at $1.18 \%$ between LBM-based simulation and the analytical result. The simulation result is shown in Fig. 9: a steady-state isotherm plot where blue indicates a low-temperature fluid and red indicates a high temperature. When the Rayleigh number is 1728 , a perturbation will grow, resulting in a rise in the convection. Hence, heat will not only propagate through conduction but also via convection. The streamline and velocity vector in Fig. 10 shows how the fluid is flowing in the system. We can see that the perturbation position becomes the position of arising fluid when convection occurs.

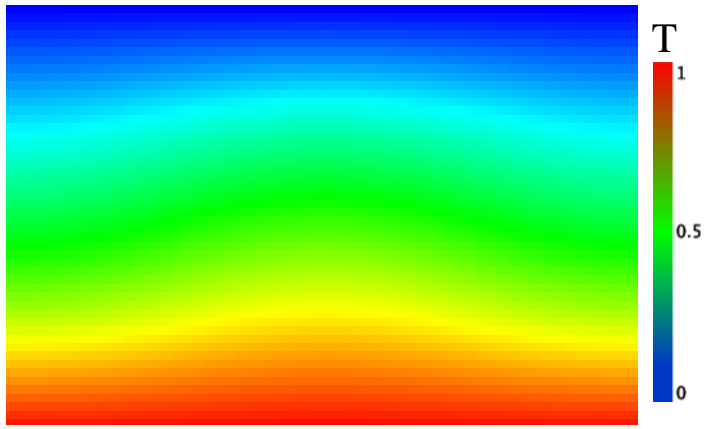

Fig. 9 Steady-state Isotherm of the natural convection when Rayleigh number is 1728

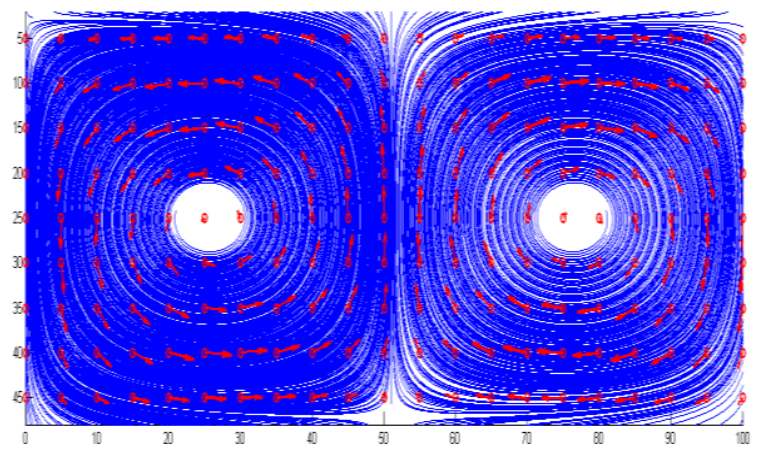

Fig. 10 Streamline and velocity vector plot of natural convection when $\boldsymbol{R a}=\mathbf{1 7 2 8}$

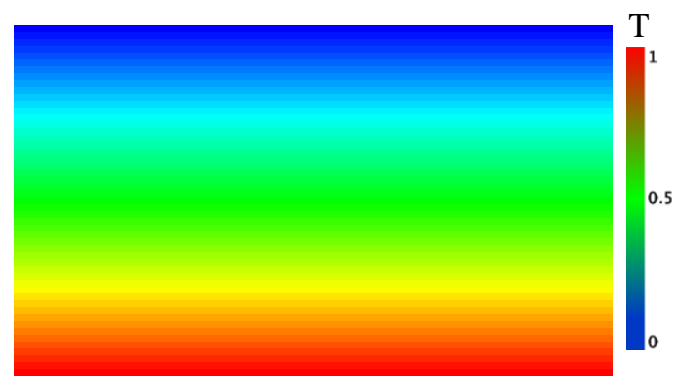

Fig.11 Steady-state isotherm with Rayleigh number under critical value.

The perturbation will decay whenever the Rayleigh number is lower than its critical value, causing no convection to be observed. In Fig. 11, the temperature changes linearly from high to low temperature because heat will only propagate through conduction. Therefore, no circulating fluid exists in the system. Or in other words, the system remains stable until it reaches a steady-state.

Furthermore, if the Rayleigh number is far above its critical value $(R a=2,000,000)$, heat propagation through convection will dominate. The perturbation will produce a plume-like phenomenon. The simulation is shown in figure 12. After it reaches the steady-state, the isotherm plot will show two vortexes that are caused by convection (Fig. 13). 

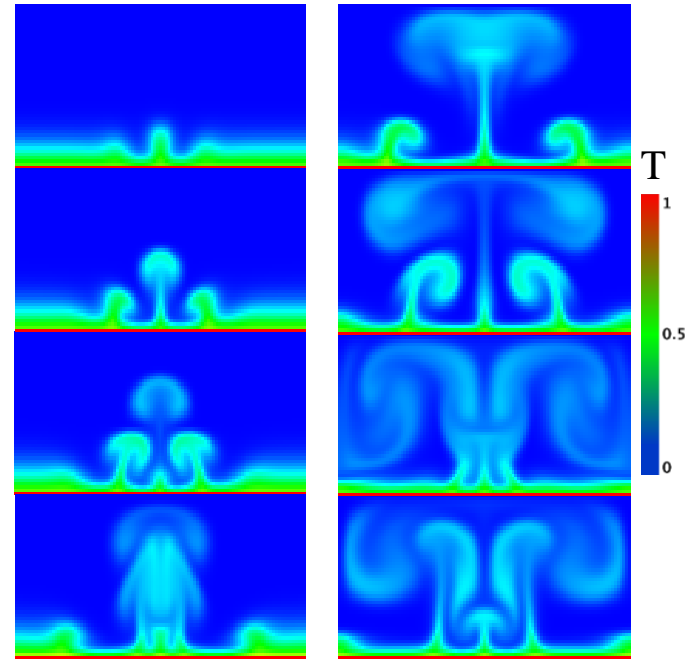

Fig. 12. Several snapshots of Isotherm plot when Rayleigh number is 2,000,000.

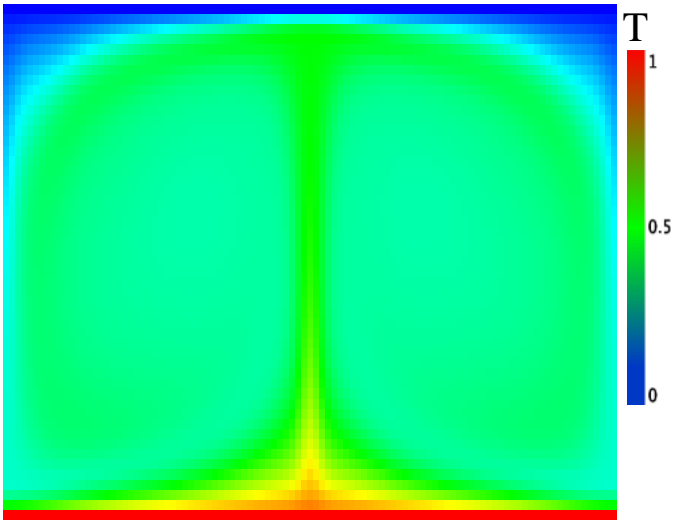

Fig. 13. Steady-state isotherm with Rayleigh number is $2,000,000$
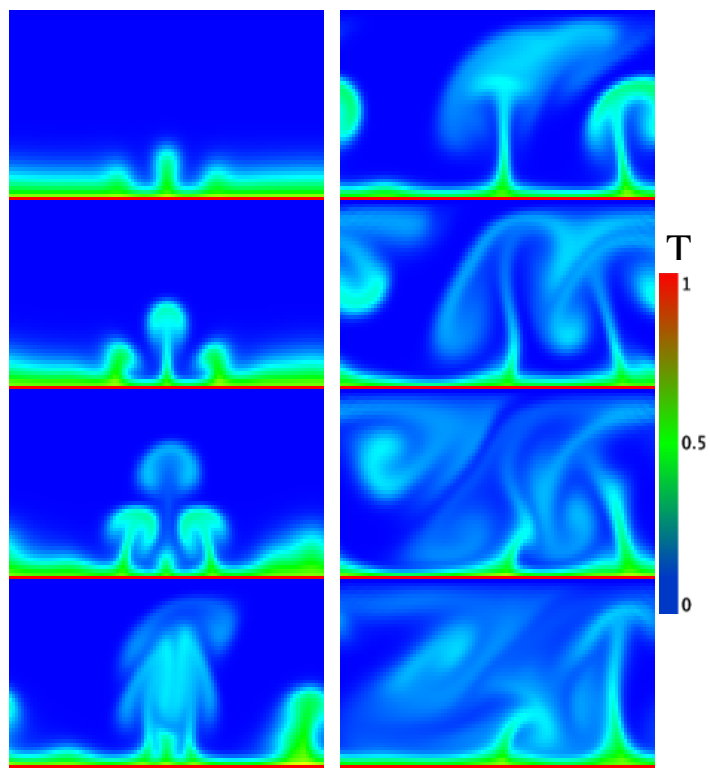

Fig. 14. Several snapshots of Isotherm plot in mantle convection simulation $(\boldsymbol{R} \boldsymbol{a}=$ $2,000,000$ ).

\section{Mantle Convection}

By combining the lid-driven cavity and natural convection model, the result as shown in Fig. 14 is obtained. We can see how the upper plate movement influences the flow of fluid. The rising fluid gets affected by the moving plate to the right side. This phenomenon is similar to mantle convection, where the plume from the bottom of the mantle arises to the upper-moving-cold tectonic plate. In Fig. 15, the plume's position is shifted from left to right. We hypothesize that this is caused by a relatively high velocity of the upper plate, simple fluid assumptions, narrow gap between plates, or periodic boundary condition effects.
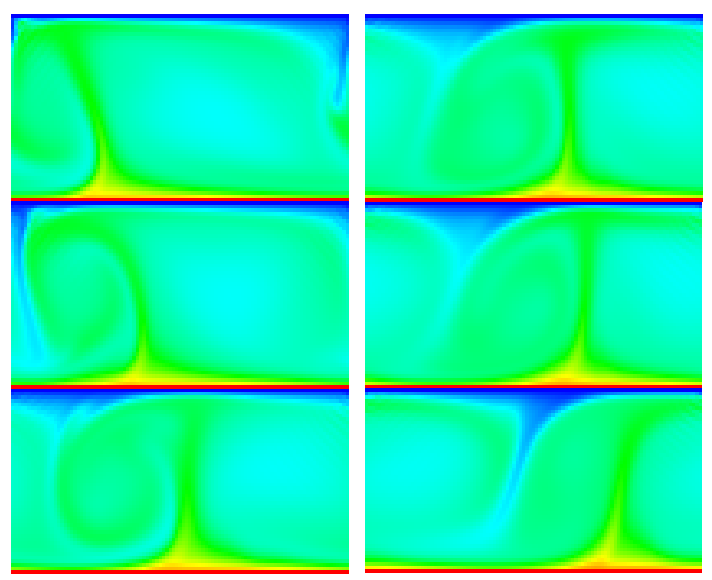

Fig. 15. Several snapshots of Isotherm plot in mantle convection simulation after $\boldsymbol{t}=\mathbf{2 9 , 0 0 0}$
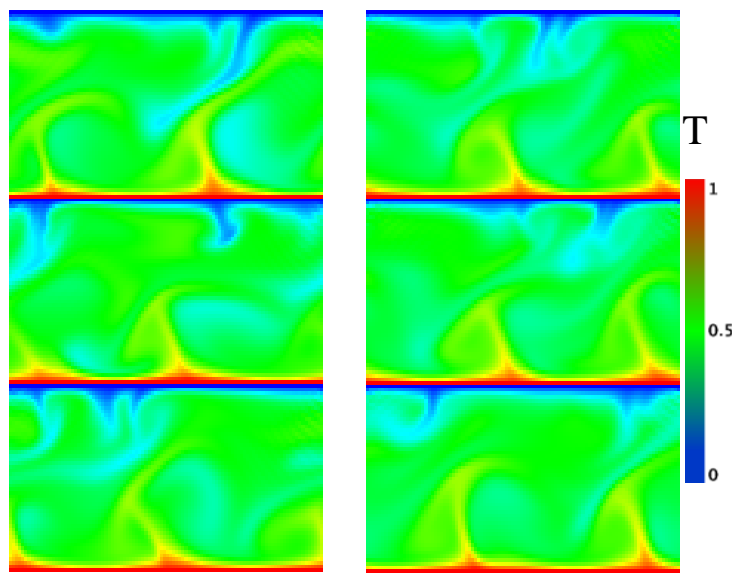

Fig. 16. Several snapshots of Isotherm plot in mantle convection simulation for $\boldsymbol{U}=\mathbf{0 . 1 2}$

In contrast with the former case, if the upper plate velocity is higher than 0.12 , the plume will shift from right to left. This is shown in figure 16 . To get an insight into how this phenomenon happens, we plot the streamline and velocity vector of both cases $(U=$ 0.1 and $U=0.12$ ). We found that shifting the plume from right to left is caused by another vortex between 
the upper plate and the lowest vortex.

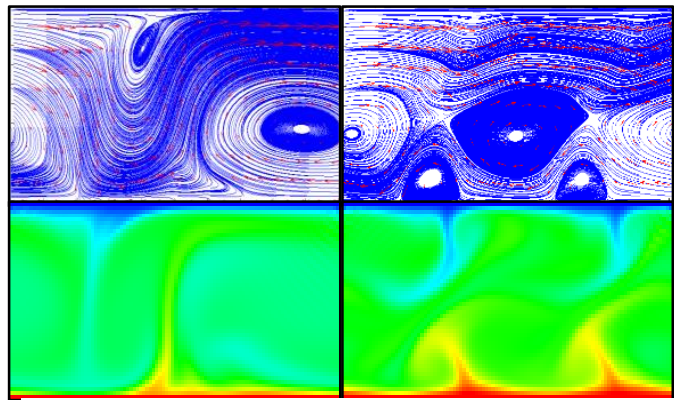

Fig. 17. Streamline comparison between upper plate velocity (left) $\boldsymbol{U}=\mathbf{0 . 1}$ and (right) $\boldsymbol{U}=$ 0.12

\section{CONCLUSION}

In this paper, we successfully simulate lid-driven cavity and natural convection using the Lattice Boltzmann Method. Even though combining these results to create mantle convection simulation gives a promising development, adjusting parameter and boundary conditions are needed to produce a more realistic state in mantle convection.

\section{REFERENCES}

[1] James E. Broadwell, Shock Structure in a Simple Discrete Velocity Gas, The Physics of Fluids 7, Issue 8, 1243, 1964.

[2] J. Hardy, O. de Pazzis, and Y. Pomeau, Molecular dynamics of a classical lattice gas: Transport properties and time correlation functions, Phys. Rev. A, Vol. 13, issue 5,pp 1949-1961, 1976.

[3] Frisch, U; d'Humieres, D; Hasslacher, B; Lallemand, P; Pomeau, Y; Rivet, J P. Lattice gas hydrodynamics in two and three dimensions. Conference: Modern approaches to large nonlinear physical systems workshop, Santa Fe, NM, USA, 27 Oct 1986.

[4] Daniel H. Rothman, Stiphane Zaleski, LatticeGas Cellular Automata: Simple Models of Complex Hydrodynamics, Cambridge University Press, 1997.

[5] Mohamad, A. A.. Lattice Boltzmann Method: Fundamentals and Engineering Applications with Computer Codes. London: Springer., 2011

[6] He, X., \& Luo, L. S.. Theory of the lattice Boltzmann method: From the Boltzmann equation to the lattice Boltzmann equation. Physical Review E, 6811-6817, 1997.

[7] Wolf-Gladrow, D. A.. Lattice-Gas Cellular Automata and Lattice Boltzmann Models: An Introduction. Germany: Springer, 2000.
[8] Guy R. McNamara and Gianluigi Zanetti, Use of the Boltzmann Equation to Simulate Lattice-Gas Automata, Phys. Rev. Lett. 61, 2332, 1988.

[9] F. J. Higuera ${ }^{1}$ and J. Jiménez, Boltzmann Approach to Lattice Gas Simulations EPL (Europhysics Letters), Volume 9, Number 7, EPL 9 663, 1989.

[10] Bhatnagar, P. L., Gross, E. P., \& Krook, M.. A Model for Collision Processes in Gases. I. Small Amplitude Processes in Charged and Neutral One-Component Systems. Physical Review, 511-525, 1954.

[11] James D. Sterling and ShiyiChen, Stability Analysis of Lattice Boltzmann Methods, Journal of Computational Physics Volume 123, Issue 1, Pages 196-206, 1996

[12] Zhaoli Guo and T. S. Zhao, A Lattice Boltzmann Model For Convection Heat Transfer In Porous Media, Numerical Heat Transfer, Part B, 47: 157-177, 2005.

[13] Jia Wanga, Donghai Wanga, Pierre Lallemand b, Li-Shi Luo, Lattice Boltzmann simulations of thermal convective flows in two dimensions, Computers and Mathematics with Applications 65, 262-286, 2013.

[14] Peter Mora and David A. Yuen, Simulation of plume dynamics by the Lattice Boltzmann Method, Geophys. J. Int., 210, 1932-1937, 2017.

[15] Renkun Dai, Qingfei Bian, Qiuwang Wang, Min Zeng, Evolution of natural convection melting inside cavity heated from different sides using enthalpy based lattice Boltzmann method, International Journal of Heat and Mass Transfer 121, 715-725, 2018.

[16] Ziliang Rui, Juan Li, Jie Ma, Han Cai, Binjian Nie, Hao Peng, Comparative study on natural convection melting in the square cavity using lattice Boltzmann method, Results in Physics 18,103274, 2020.

[17] Abderrahmane Bourada, Asma Ouahouah, Kaoutar Bouarnouna, Karim Ragui1, Abdelkader Boutra1, 2, and Youb Khaled Benkahla1, Multiple-relaxation-time Lattice Boltzmann model for flow and convective heat transfer in lid-driven cavity with a porous obstacle, MATEC Web of Conferences 330, 01007, 2020.

[18] Becker, T. W., Global plate kinematics and dynamical models of the mantle, American Geophysical Union, Fall Meeting 2018.

[19] Ghia, U., Ghia, K. N., \& Shin, C. T. (1982). 
High-Re Solutions for Incompressible Flow Using the Navier-Stokes Equations and a Multigrid Method. Journal of Computational Physics, 387-411.

[20] Chandrasekhar, S. (1961). Hydrodynamic and Hydromagnetic Stability. London: Oxford University Press. 